\title{
The improvement of the aluminum alloy 6061 surface hydrophobic property by using wire cut electrical discharge machining
}

\author{
Yanling Wan ${ }^{1, \text { a }}$, Zhigang Liü ${ }^{2, \mathrm{~b}}$ Zhongxu Lian ${ }^{3, \mathrm{c}}$ Huadong Yu ${ }^{4, \mathrm{~d}^{*}}$ Kui Xia ${ }^{5, \mathrm{e}}$ \\ ${ }_{1,2,3,4,5}$ College of Mechanical and Electric Engineering, Changchun University of Science and \\ Technology, Changchun, Jilin Province, China \\ awanyanling@cust.edu.cn, ${ }^{\mathrm{a}}$ liuzhigangmeng@163.com, ${ }^{\mathrm{d}}$ yuhuadong@cust.edu.cn
}

\begin{abstract}
Keywords: Hydrophobic WEDM Contact angle Processing parameters Micro-nano structures Abstract. To improve the hydrophobic properties of the metal substrate and the using range, wire cut electrical discharge machining(WEDM) processes aluminum alloy 6061 plane twice in this paper. Under electrical discharge machining for the first time in the processing parameters of the pulse width $12 \mu \mathrm{s}$, pulse interval $84 \mu \mathrm{s}$, without the low surface energy materials, the static contact angles improve from $76.5^{\circ}$ on the smooth aluminum alloy plane to $112.5^{\circ}$, reaching the hydrophobic state. On the basis of the processing, WEDM under 10 groups of different machining parameters processes the aluminum alloy plane for the second time, the static contact angel of up to $153.1^{\circ}$, achieving super-hydrophobic state. The aluminum alloy surfaces processed have micron grade similar pit craters with nanoscale structures under scanning electron microscopy(SEM). This kind of micro-nano structures make the aluminum alloy surface have the hydrophobic and super-hydrophobic properties.
\end{abstract}

\section{Introduction}

Since the discovery of "lotus effect", Superhydrophobic surface refers to the water droplets on the sold surface contact angle of $150^{\circ}$ above. There are a lot of super-hydrophobic phenomena in nature. The static contact angle of the water droplets on the surface of lotus leafs, rice leafs red rose petals, water strider legs, butterfly wings, fish scales et al.[1] are greater than $150^{\circ}$, showing the superhydrophobic property. The water droplets are easy to tumble from the surface, because the areas of the surface in contact with the water droplets are very small, so the super-hydrophobic surface has the self-cleaning, anti-corrsion, waterproof, anti-stiction pollution prevention and other functions[2-5].Based on the super-hydrophobic having the broad application prospects, nowadays more and more experts and scholars at home and abroad pay attention to the preparation and usage of the superhydrophobic surface. Jiang Lei et al.[6] used polystyrene dimethyl acrylamide solution to prepare miecr/nano coarse structures of super hydrophobic surface of polystyrene through the electrospinning method, which made the static contact angle be $160^{\circ}$. Liao et al.[7] took the method of layer-by-layer self-assembly with aminated multiwall carbon(MWNT) to obtain the materials with superhydrophobic property. Gurav et al.[8,9] prepared the transparent super-hydrophobic surface at room temperature by using soi-gel process. Lee et al.[10] let nickel take place of polymer to prepare imitation bamboo surface of super-hydrophobic surface through the template printing method. Zhang et al.[11] stretched Teflon membrane to get the superhydrophobic surface with a large number of nanoscale hole.

Chang Chun University of Science and Technology[12] used WEDM to machine sub-millimeter-scale groove structure in the aluminum alloy surface to reach super-hydrophobic state. Hower, due to the directional groove structure, the static contact angle was $158^{\circ}$ in the direction perpendicular to the grooves, and the static contact angle was $145^{\circ}$ in the direction parallel to the grooves. This paper also adopts WEDM to process the aluminum alloy plane. Due to the uniform discharge between wire electrode and workpiece in the process, the surface structures are not directional, so the static contact angles of water droplets on the surface are not directonal. WEDM processes aluminum alloy plane twice to construct micro-nano structures, improving the surface contact angle up to the superhydrophobic effect. 


\section{The test method}

This article mainly uses the high-speed WEDM(DK7732) for the preparation and improvement of the hydrophobic property of aluminum alloy 6061 surface. The processing steps are as follows: In the processing parameters of the pulse width $12 \mu \mathrm{s}$, pulse interval $84 \mu \mathrm{s}$, WEDM preliminary processed aluminum alloy plane; then changing the processing parameters to process the plane processed for the first time so as to study the influence of the different processing parameters on the wettability of aluminum alloy plane(the ratio of between pulse width and pulse interval is $1 / 7$ ), the specific processing parameters such as Table 1:

\begin{tabular}{|c|r|r|r|r|r|r|r|r|r|r|}
\hline Test-piece & & 2 & 3 & 4 & 5 & 6 & 7 & 8 & 9 & 10 \\
\hline Parameters & & & & & & & & & & \\
\hline Pulse width & 32 & 24 & 16 & 12 & 8 & 6 & 4 & 3 & 2 & 1 \\
\hline Pulse interval & 224 & 168 & 114 & 84 & 56 & 42 & 28 & 21 & 14 & 7 \\
\hline
\end{tabular}

The test-pieces after processed were cleaned by ultrasonic cleaning machine in acetone, alcohol, deionezed water for 5 minutes, then air-drying. JCJ-001 Contact Angle Measurement Instrument measured the static contact angles of the test-piece surfaces with $5 \mu 1$ water drop, as showed in Fig. 1.

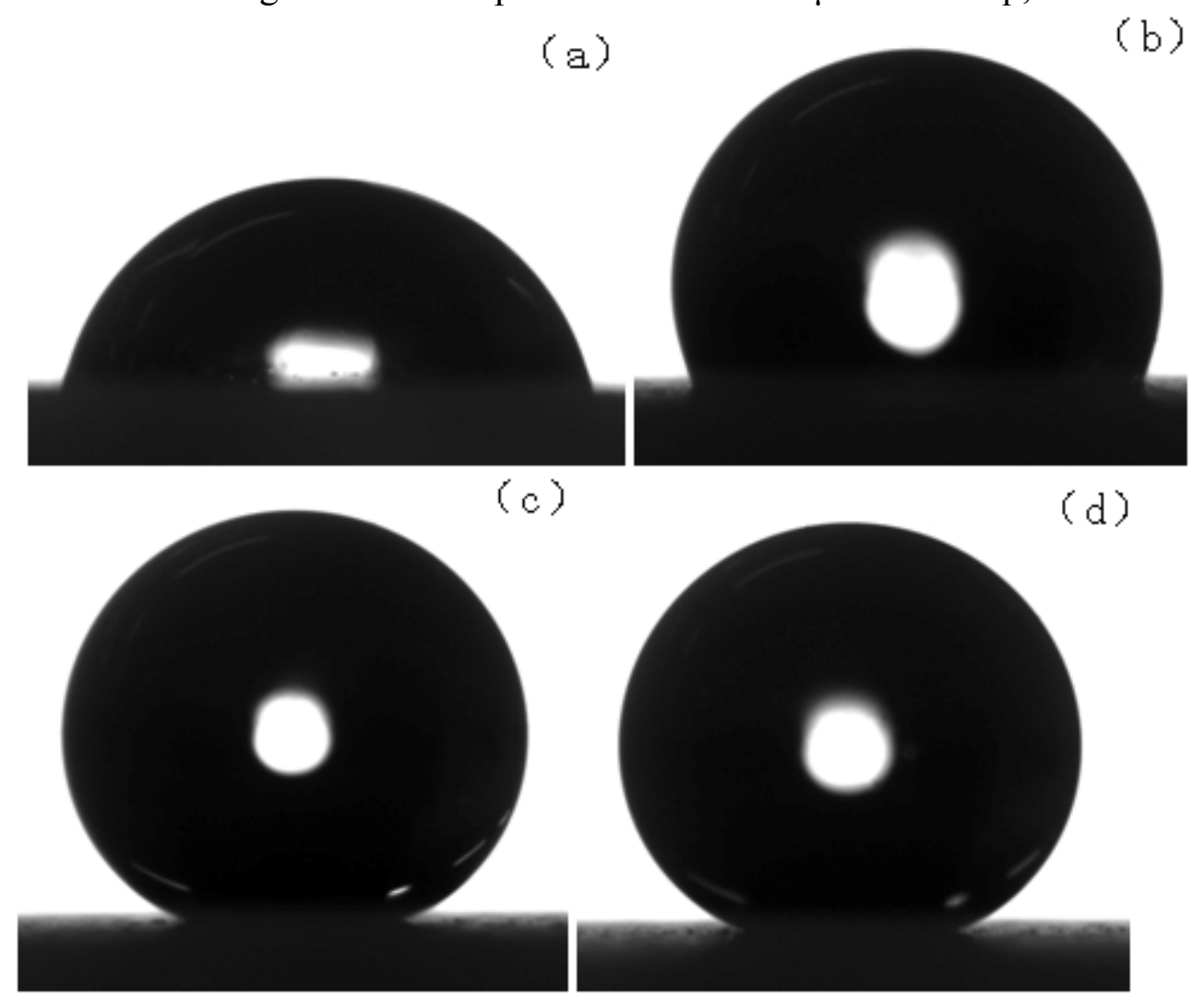

Fig. 1 The image of the static contact angle on different surfaces (a) the smooth surface (b) the surface processed for the first time (c) the surface of the first test-piece (d) the surface of the ninth test-piece

\section{Test analysis}

After the aluminum alloy were processed by WEDM for the first time, the static contact angles of water droplets improved from $76.5^{\circ}$ on the smooth aluminum alloy plane to $112.5^{\circ}$ (Fig. $\left.1 \mathrm{a}, \mathrm{b}\right)$. The static contact angles of test-pieces are shown in Fig. 2 


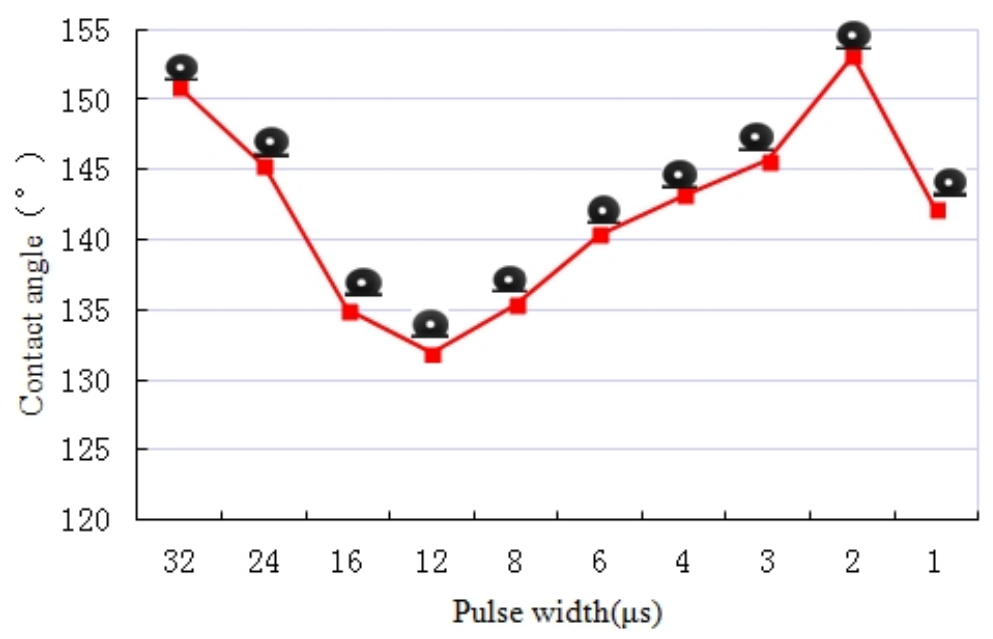

Fig 2 The summary line chart of the static contact angle

Observe the aluminum alloy surface microstructures in FEI Quanta 250 FEG environmental SEM, as shown in Fig. 3.

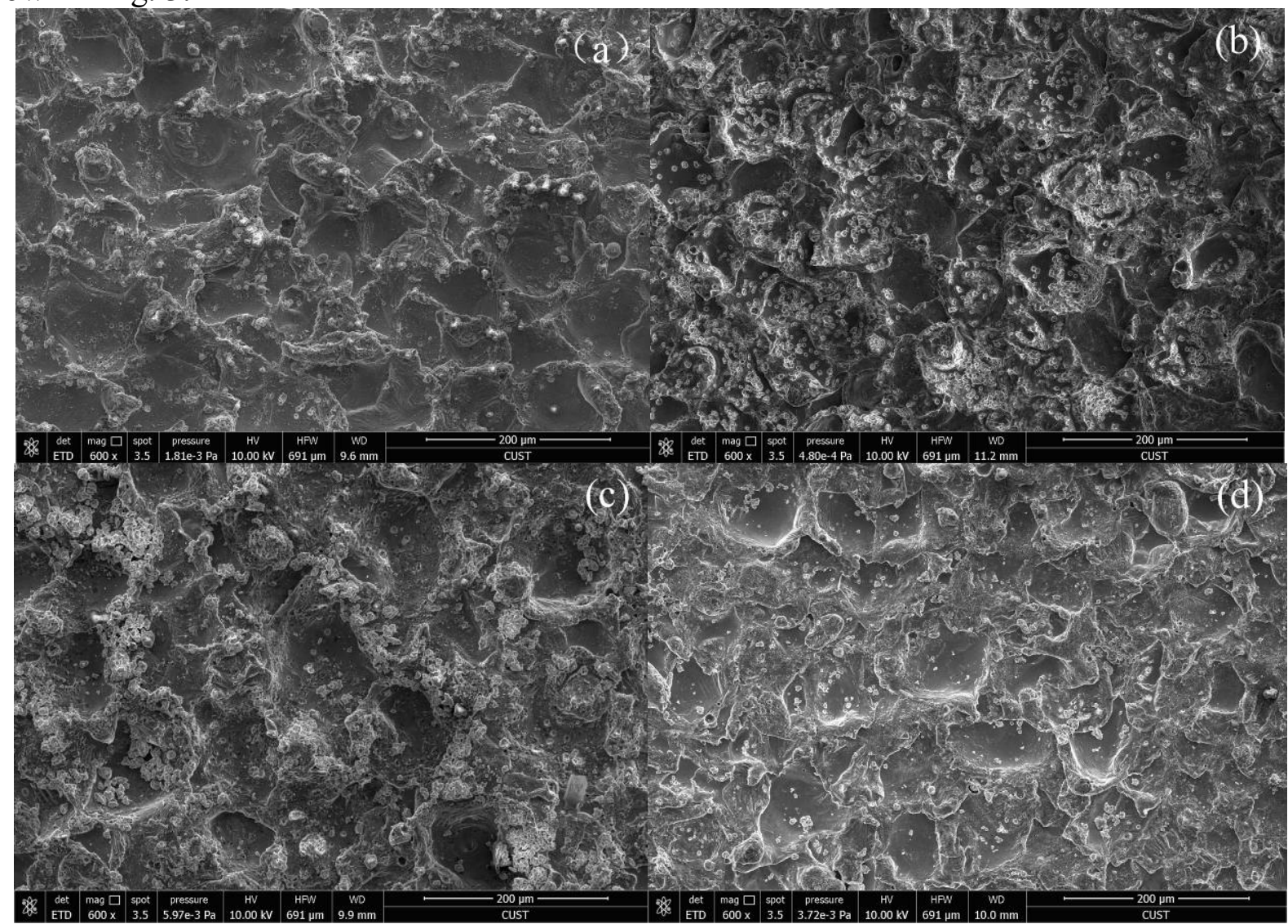

Fig. 3 SEM image of different surfaces (a) the surface processed for the first time (b) the surface of the forth test-piece (c) the surface of the first test-piece (d) the surface of the ninth test-piece

Fig. 3b,c,d comparing with Fig 3a, the micro-nano structures on former surface are less than the latter on account of WEDM process[13]. When WEDM works, there is a plasma channel between wire electrode and workpiece due to the role of the strong electric field, where electronics with a high kinetic energy bombard the workpiece, resulting in that the surface of workpieces form micron grade similar craters, which the size of are decided by the pulse width of electric sparkle. And splashes of liquid metal condense into nanoscale structures on their surfaces.

WEDM processed aluminum alloy plane twice to construct micro-nano composit structures similar to the lotus leaf. These micro-nano composite structures retained the air between the water droplets and aluminum alloy plane, forming a layer of "air cushion" to make the water droplets not completely dip into the aluminum alloy surface, thus increasing the water contact angle. In the test-pieces from 1 to 
4, the static contact angle on the surface of the aluminum alloy gradually decreased with the decrease of the pulse width, and the static contact angle of the forth test-piece is minimal,131.9 $9^{\circ}$ The reason is that in the first 4 set of test-pieces, the second processing pulse width was greater than the first one, destroying the micron grade structures constructed for the first time(Fig. 3c), however, increasing the nanoscale structures which increased with the decrease of the pulse width. In the test-pieces from 5 to 9, the static contact angle gradually increased with the decrease of the pulse width, and the static contact angle of the ninth test-piece was maximum, 153.1 ${ }^{\circ}$. The reason was that in these 5 test-pieces, the second processing pulse width was smaller than the first one, constructing the smaller micron grade structures on the micron structures built for the first time(Fig.3d), and increasing the nanoscale structures, consequently resulting in increasing the static contact angle. When the tenth piece-test was processed, the second processing pulse width was far less than first one, hardly having effect on the surface processed for the first time., which maked the static contact angle be smaller than one of the ninth test-piece.

\section{Conclusions}

(1)WEDM processed aluminum alloys plane twice to improve the hydrophobic property of their surfaces.

(2)WEDM, under 10 groups of different processing parameters, processed the aluminum alloy planes for the second time, which were processed in the processing parameters of the pulse width $12 \mu \mathrm{s}$, pulse interval $84 \mu \mathrm{s}$. The static contact angle on the surface of the aluminum alloy gradually decreased with the decrease of the pulse width, the static contact angle minimum $131.9^{\circ}$ in the pulse width of $12 \mu \mathrm{s}$, then gradually increased with the decrease of the pulse width, the static contact angle minimum $153.1^{\circ}$ in the pulse width of $2 \mu \mathrm{s}$.

(3)WEDM processed aluminum alloy planes to construct the micro-nona composite structures which maked aluminum alloy surfaces with the superhydrophobic properties.

\section{Acknowledgment}

This work was supported by the National Natural Science Foundation of China (51275056), and the Jilin Province Science and Technology Development Program of China (Grant No. 20150204018GX).

\section{References}

[1] Kesong Liu, Lei Jiang, Bio-inspired design of multiscale structures for function integration, Nano Today (2011)6,155-175.1

[2] Barthlott W,Neinhuis C, Purity of the sacred lotus, or escape from contaimination in biological surfaces.Planta, 1997, 202(1):1-8.

[3] Nakajima A, Fujishima A, Hashimoto K, et al, Preparation of transparent superhydrophobic boehmite and silica films by sublimation of aluminum acetylacetonate, Adv Mater,1999,11(16):1365-1368.

[4] Nakajima A, Design of transparent hydrophobic coating, J Ceram Soc Jpn,2004, 112(10):533-540. [5] Zhang H Lamb R, Lewis J, Engineering nanoscale roughness on hydrophobic surface-preliminary assessment of fouling behavior, Sci Technol Adv Mat, 2005,6(3-4):236-1239.

[6] L. Jiang, Y. Zhao, J. Zhai, A Lotus-leaf-like superhydrophobic surface: A porous microsphere/nanofiber composite film prepared by electrohydro-dynamics, Angew Chem Int Ed, 2004(43):4338-4341.

[7] K.S. Liao, A. Wan, J.D. Batteas, D.E. Bergbreiter, Superhydrophobic surfaces formed using layer-by-layer self-assembly with aminated multiwall carbon nanotubes,Langmuir,24(2008):4245-4253.

[8] A.B. Gurav, S.S. Latthe, C. Kappenstein, S.K. Mukherjee, A.V. Rao, R.S. Vhatkar, Porous water repellent silica coating on glass by sol-gel method, Journal of Porous Mater, 18(2011):361-367. 
[9] S.A. Mahadik, M.S. Kavale, S.K. Mukherjee, A.V Rao, Transparent superhydrophobic silica coatings on glass by sol-gel method, Applied Surface Science, 257(2010): 333-339.

[10] S.M. Lee, T.H Kwon, Mass-producible replication of highly hydrophobic surfaces from plant leaves, Nanotechnology, 2006(17): 3189-3196.

[11] J.L. Zhang, Y C. Han, Superhydrophobic PTFE surfaces by extension. Macromol Rapid Common, 2004(25): 1105-1108.

[12] Yan-Ling Wan,Jun Lou et al.Single-step fabrication of bionic-superhydrophobic surface using reciprocating-type high-speed wire cut electrical discharge machining, China. Sci. Bull. (2014) 59(28):3691-3695.

[13] Guofeng Cao, Electrical discharge machining technology, Beijing: Chemical industry press, 2004.8:12-14(In Chinese). 